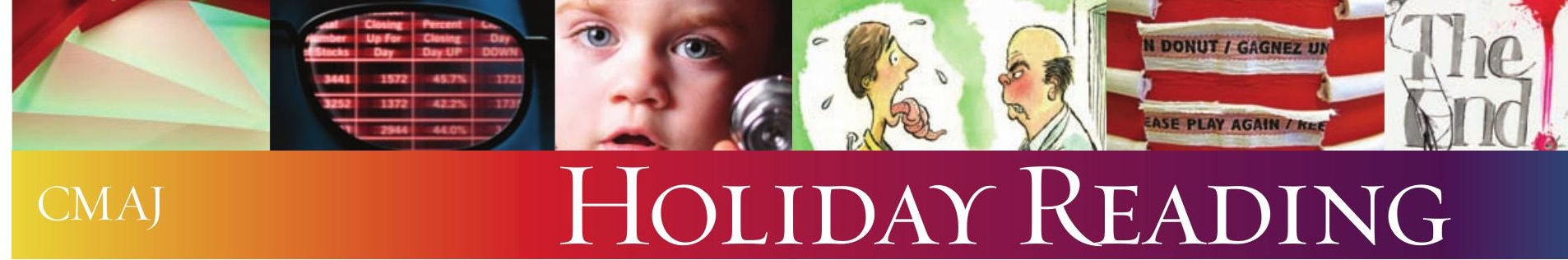

RESEARCH OF A HOLIDAY KIND

\title{
Elevators or stairs?
}

\author{
Sachin Shah MD, Michael O'Byrne MD, Merne Wilson RN MSc, Thomas Wilson MD
}

\begin{abstract}
Background: Staff in hospitals frequently travel between floors and choose between taking the stairs or elevator. We compared the time savings with these two options.

Methods: Four people aged 26-67 years completed 14 trips ranging from one to six floors, both ascending and descending. We compared the amount of time per floor travelled by stairs and by two banks of elevators. Participants reported their fatigue levels using a modified Borg scale. We performed two-way analysis of variance to compare the log-transformed data, with participant and time of day as independent variables.

Results: The mean time taken to travel between each floor was 13.1 (standard deviation [SD] 1.7) seconds by stairs and 37.5 (SD 19.0) and 35.6 (SD 23.1) seconds by the two elevators $(F=8.61, p<0.001)$. The difference in time taken to travel by stairs and elevator equaled about 15 minutes a day. Self-reported fatigue was less than 13 (out of 20) on the Borg scale for all participants, and they all stated that they were able to continue their duties without resting. The extra time associated with elevator use was because of waiting for its arrival. There was a difference in the amount of time taken to travel by elevator depending on the time of day and day of the week.

Interpretation: Taking the stairs rather than the elevator saved about 15 minutes each workday. This $3 \%$ savings per workday could translate into improved productivity as well as increased fitness.
\end{abstract}

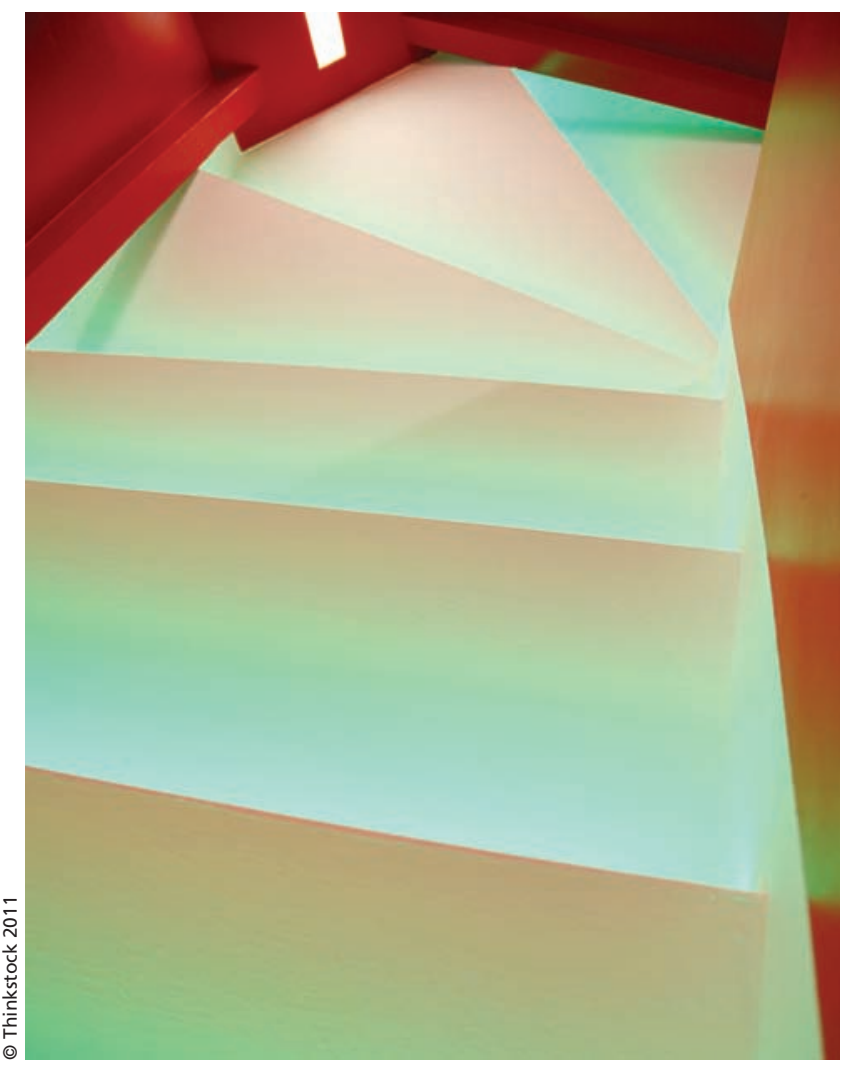

$\mathrm{T}$ he areas visited by hospital staff in internal medicine are often on different floors, with staff making multiple trips by stairs or elevator each day. The reasons for choosing to take the elevator over the stairs include concerns about dyspnea and fatigue, and the amount of time saved.

Although many people feel that taking the elevator saves time, there is little evidence to support this claim. We compared travel times for staff taking elevators and stairs in a typical urban hospital.

\section{Methods}

Two male residents (aged 26 and 30 years) and two faculty members (a 56-year-old woman and a 67-year-old man) par- ticipated in this study. None of the participants had cardiovascular, respiratory or mobility problems.

The Royal University Hospital is a 391-bed tertiary care centre in Saskatoon, Saskatchewan. The seven-storey hospital has three banks of two elevators each, one near the medical imaging department ("x-ray elevators") and two in the outpatient area of the main floor ("outpatient elevators"). There are stairways located near each set of elevators, with 18 steps between each floor.

We measured the amount of time that each participant took to walk 14 routes (ground floor to main, ground to third, ground to fourth, ground to sixth, main to third, main to fourth and main to sixth; ascending and descending). We recorded the amount of time from elevator door to elevator door on the 
designated level for both types of travel. The participants walked during all trips and did not "rush." They recorded their subjective fatigue levels using a modified Borg scale. ${ }^{1}$

The same participants completed the elevator trips during six periods: 0700-0900, 0901-1700 and 1701-2100 during the workweek and on Saturday or Sunday. We measured the amount of time elapsed from when the elevator button was pushed to arrival on the designated floor. We also recorded the time between pushing the button and when the doors opened (waiting time) and the time between when the doors opened at boarding to when the doors opened at the destination (travel time).

\section{Statistical analysis}

We assumed that there would be no more than a $10 \%$ difference between elevators and stairs in the amount of time per floor travelled. We also assumed that the standard deviation (SD) for each would be $10 \%$. We included 56 walking trips and 336 elevator trips, giving us a more than $95 \%$ power to detect this difference.

Our main outcome was time elapsed per floor travelled. The independent variables were participant and time of day. We performed two-way analysis of variance using log-transformed time data, and Duncan post-hoc testing.

\section{Results}

All walking and elevator trips were performed without incident. Participant fatigue varied from 6 (light) to 13 (moderately heavy) on a modified Borg scale. There was no significant difference in fatigue between participants. All participants felt that they could continue their daily activities immediately after every trip.

The mean time elapsed per floor was 13.1 (SD 1.7) seconds by taking the stairs, 37.5 (SD 19.0) seconds by taking the outpatient elevators and 35.6 (SD 23.1) seconds by taking the $\mathrm{x}$-ray elevators. The difference in time between the stairs and

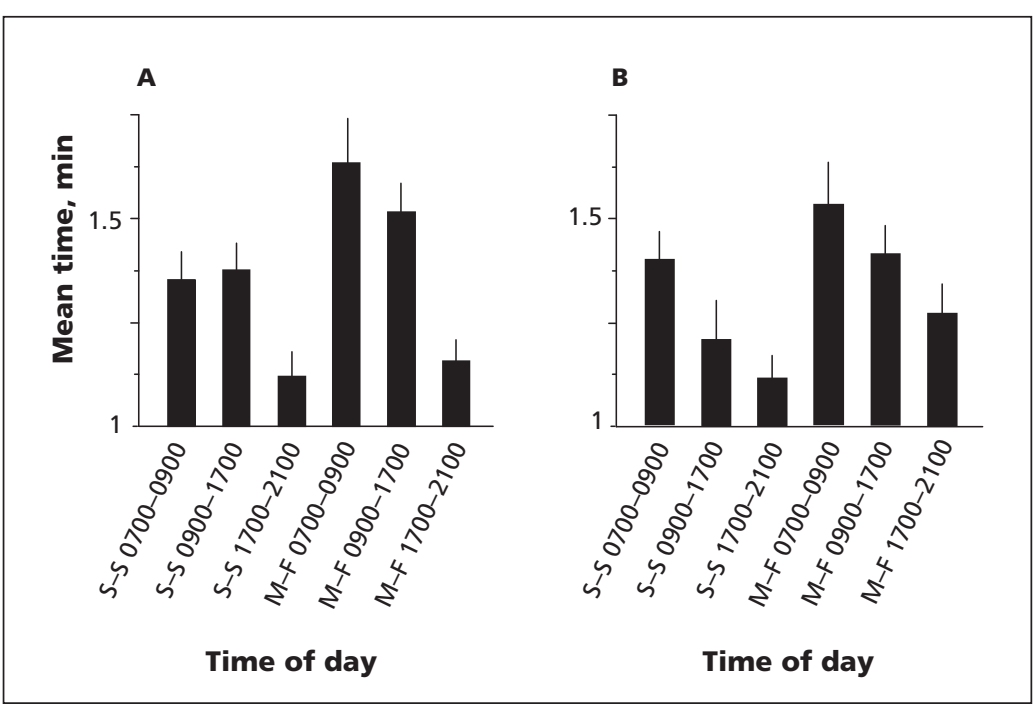

Figure 1: Mean length of time per trip by (A) "outpatient" elevator and (B) "x-ray" elevator at different times. Note: $\mathbf{S}-\mathbf{S}=$ Saturday and Sunday; $\mathbf{M}-\mathbf{F}=$ Monday through Friday. Error bars represent standard deviations. either bank of elevators was significant $(p<0.001)$. There was no significant difference between participants or between ascending or descending trips by stairs.

The elapsed time for the longest trip by stairs (six floors) was between 58 and 92 seconds. There was no difference between participants.

The mean elapsed total time for 14 trips was 10.3 (SD 0.2) minutes by stairs and 24.6 (SD 0.4) minutes and 19.6 (SD 0.4) minutes by the outpatient and $\mathrm{x}$-ray elevators, respectively. There was no difference in time between the two elevator banks.

The amount of time taken to travel by elevator varied with time and day of the week (Figure 1). The average amount of time spent waiting for and travelling by elevator varied from 1.0 (SD 0.1) minutes for weekend trips in the early morning to 1.8 (SD 0.2) minutes for trips during the same time on weekdays. On weekdays from 0700-0900, the time taken to travel six floors via elevator varied from 1.1 to 6.9 minutes. Taking the elevator took longer than the stairs even during the early morning period on weekends (1.0 [SD 0.1] v. 0.7 [SD 0.1] minutes; $p$ $<0.02$ ). These differences were almost entirely because of the time spent waiting for the elevator (data not shown).

\section{Interpretation}

We found that taking the stairs rather than the elevator saved almost 15 minutes each day for each of the participants, who were typical members of the Department of Medicine. If we assume that an average workday is eight hours, this represents a 3\% savings. Compare this with the $1.0 \%-1.4 \%$ gains in productivity because of technology improvements in the past 20 years. ${ }^{2}$ The 15 minutes saved by each participant was not realized at the cost of undue fatigue.

A $3 \%$ time savings may seem trivial. However, when it is placed in the context of a typical day in the hospital, it becomes more significant. For example, Tipping and colleagues found that hospital physicians spent only $19 \%$ of their day providing direct patient care. ${ }^{3}$

There are few studies that we can compare with ours. Westmeier-Shuh and colleagues found that university students took twice as long to ascend or descend one floor by elevator compared with stairs (17.4 and 15.8 seconds v. 34.1 and 37.6, respectively). ${ }^{4}$ Their estimates of the time per floor are very close to ours. Their study did not include longer trips.

The IBM Smarter Building survey in 2010 found that office workers in US cities waited for and travelled in elevators an average of 3.2-4.2 minutes each day. ${ }^{5}$ This is much shorter than our estimate of total elevator time of 20-25 minutes. However, few office workers would take 14 trips per day, as is common with hospital staff.

Meyer and colleagues found that encouraging sedentary office workers to use the stairs resulted in an increase from 4.5 to 20.6 flights travelled per workday. ${ }^{6}$ After six months, there was a $6 \%$ increase in aerobic capacity and substantial improvements in body fat and lipid markers. The 
14 trips included in our study represent about 48 flights per day, which is double that in the study by Meyers and colleagues. ${ }^{6}$

\section{Limitations}

Our study has limitations, including the small sample size. However, the participants were not trained athletes, and their age range is representative of the department staff's age range. Our building has just seven storeys, and our building has no express or staff-dedicated elevators. It is possible that the results would be different in buildings with different characteristics. However, given the significant differences in time between taking the stairs and taking the elevators during "off" hours (Saturday and Sunday 1700-2100), we believe that, in most cases, taking the stairs would save time.

We assumed that time waiting for and travelling in an elevator was "wasted." It is possible that some staff could use this time to consult colleagues or electronic information sources.

\section{Conclusion}

Taking the stairs rather than the elevator saved about 15 minutes each workday This, plus the benefit of increased fitness, should convince hospital staff to take the stairs whenever possible.

\section{References}

1. US Centers for Disease Control and Prevention. Perceived exertion (Borg Rating of Perceived Exertion Scale). Atlanta (GA): The Centers; 2011. Available: www.cdc.gov/physicalactivity/everyone/measuring/exertion.html (accessed 2011 June 9).

2. Organisation for Economic Co-operation and Development. Measuring productivity: measurement of aggregate and industry-level productivity growth. Paris (France): The Organisation; 2001. Available: www.oecd.org/dataoecd/59/29 /2352458.pdf (accessed 2011 June 9).

3. Tipping MD, Forth VE, O'Leary KJ, et al. Where did the day go? A time-motion study of hospitalists. J Hosp Med 2010;5:323-8.

4. Westmeier-Shuh J, Parr P, DeWitt C, et al. Time required for stair and elevator use: implications for a physical activity intervention. Med Sci Sports Exerc 2011;39:S195.

5. IBM. Smarter buildings survey: consumers rank their office buildings. Armonk (NY): IBM; 2010. Available: www-03.ibm.com/press/attachments/IBM_Smarter _Buildings_Survey_White_Paper.pdf (accessed 2011 June 9).

6. Meyer P, Kayser B, Kossovsky MP, et al. Stairs instead of elevators at workplace: cardioprotective effects of a pragmatic intervention. Eur J Cardiovasc Prev Rehabil 2010;17:569-75.

Competing interests: None declared.

Correspondence to: Dr. Thomas Wilson, thomas.wilson@usask.ca

Affiliations: From the Department of Medicine, University of Saskatchewan, Saskatoon, Sask.

Contributors: Sachin Shah conceived the study design. All of the authors contributed to the design, performance and analysis of the study. All of the authors revised it for intellectual content and approved the final version submitted for publication.

CMAJ 2011. DOI:10.1503/cmaj.110961 